\title{
Kinetics of a Diffusive Capture Process: Lamb Besieged by a Pride of Lions
}

\author{
P. L. Krapivsky ${ }^{1}$ and S. Redner ${ }^{2}$ \\ ${ }^{1}$ Courant Institute of Mathematical Sciences \\ New York University, New York, NY 10012-1185 \\ ${ }^{2}$ Center for Polymer Studies and Department of Physics \\ Boston University, Boston, MA 02215
}

\begin{abstract}
The survival probability, $S_{N}(t)$, of a diffusing prey ("lamb") in the proximity of $N$ diffusing predators (a "pride of lions") in one dimension is investigated. When the lions are all to one side of the lamb, the survival probability decays as a non-universal power law, $S_{N}(t) \propto t^{-\beta_{N}}$, with the decay exponent $\beta_{N}$ proportional to $\ln N$. The crossover behavior as a function of the relative diffusivities of the lions and the lamb is also discussed. When $N \rightarrow \infty$, the lamb survival probability exhibits a log-normal decay, $S_{\infty}(t) \propto \exp \left(-\ln ^{2} t\right)$.
\end{abstract}

\section{INTRODUCTION}

Consider a particle system which consists of a diffusing prey and $N$ independent, diffusing predators, with $N$ either finite or infinite. The prey is absorbed, or dies, whenever it is touched by any of the predators. We are interested in the probability $S_{N}(t)$ for this "lamb" to survive until time $t$ when it is besieged by these predatory "lions" [1]. While this appears to be a simple problem, there are important aspects of the long-time behavior which are incompletely understood. Their resolution has fundamental ramifications for diffusive processes in the presence of complex absorbing boundaries and also practical implications, as this type of capture process appears in a variety of applications, such as diffusion-controlled chemical kinetics, wetting, melting, and commensurateincommensurate transitions (see, e. g., Refs. [2-4]). It is known rigorously that for spatial dimension $d \geq 3$, the capture process is "unsuccessful" (in the terminology of Ref. 1), in that there is a finite probability for the lamb the survive indefinitely for any $N$ and for any initial spatial distribution of the lions. This result is a consequence of the transience of random walks for $d>2$ [5]. For $d=2$, the capture process is "successful" - the lamb dies with probability one. However, diffusing lions in two dimensions are still sufficiently poor predators that the average lifetime of the lamb is infinite. Moreover, $S_{N}(t) \propto\left[S_{1}(t)\right]^{N} \propto[\ln t]^{-N}$, $i$. e., the many-body nature of the capture process is basically irrelevant.

In one dimension, however, diffusing lions are more efficient in their predation because of the recurrence of random walks [5]. This typically leads to a lamb survival probability 
which decays as a power law in time. More generally, we may consider the survival probability, $S_{N, M}(t)=S_{M, N}(t)$, when initially $N$ lions are placed to the left and $M$ lions are placed to the right of the lamb. The most interesting situation is when initially $N$ lions are to one side of the lamb. For this initial condition, realizations in which the lamb runs away from the lions leads to an anomalously slow decay of the lamb survival probability. Our primary result is to determine the asymptotic behavior of the lamb survival probability in this predatory environment, $S_{N, 0}(t) \equiv S_{N}(t)$. For finite $N$, we predict that $S_{N}(t) \sim t^{-\beta_{N}}$ with $\beta_{N} \propto \ln N$, while $S_{\infty}(t) \propto \exp \left(-\ln ^{2} t\right)$ for $N \rightarrow \infty$. We shall argue that these dependences arise from the fact that the motion of "closest" lion (whose individual identity can change with time) is enhanced compared to normal diffusion. When the number of lions is finite, this enhancement manifests itself in the diffusivity of the last lion being proportional to $\ln N$. This factor is ultimately responsible for the logarithmic increase of $\beta_{N}$ on $N$. In the limit $N \rightarrow \infty$, the co-ordinate of the last lion actually varies as $\sqrt{t \ln t}$, and leads to the lamb survival probability varying as $\exp \left(-\ln ^{2} t\right)$.

To provide context for our results, consider first a system consisting of one lamb and one lion, i. e., $(M, N)=(1,0)$ or $(0,1)$. The survival probability is trivially calculable in this case, since the distance between the lion and the lamb undergoes pure diffusion with an associated diffusivity $D_{L}+D_{\ell}$. Here $D_{L}$ and $D_{\ell}$ are the lion and lamb diffusivities, respectively. Because of the equivalence to diffusion, the survival probability is [5]

$$
S_{1,0}(t) \sim \frac{x_{0}}{\sqrt{\left(D_{L}+D_{\ell}\right) t}},
$$

where $x_{0}$ is the initial separation between the lion and the lamb. A more interesting situation is that of two lions with either: (i) one lion on either side of the lamb ("trapped" lamb), or (ii) both lions to one side ("chased" lamb). These two systems can be straightforwardly solved when the diffusivities of all three particles are different. For the trapped lamb, denote the particle positions as $x_{1}, x_{2}$, and $x_{3}$, with 1 and 3 referring to the surrounding lions, and 2 to the trapped lamb. Let the corresponding diffusivities be $D_{1}, D_{2}$, and $D_{3}$. To solve for the survival probability, it is convenient to introduce the rescaled co-ordinates $y_{i}=x_{i} / \sqrt{D_{i}}$, each of which diffuses at the same rate. The survival of the lamb corresponds to the constraints $y_{1} \sqrt{D_{1}}<y_{2} \sqrt{D_{2}}$ and $y_{2} \sqrt{D_{2}}<y_{3} \sqrt{D_{3}}$. Since the co-ordinates $y_{i}$ diffuse isotropically, lamb survival is equivalent to the survival of a random walk in three-dimensional space within the wedge-shaped region bounded by the absorbing planes $y_{1} \sqrt{D_{1}}=y_{2} \sqrt{D_{2}}$ and $y_{2} \sqrt{D_{2}}=y_{3} \sqrt{D_{3}}$. By straightforward geometric considerations [2], this three-dimensional diffusion process is equivalent to diffusion in two dimensions within a wedge of opening angle

$$
\theta=\cos ^{-1}\left(\frac{D_{2}}{\sqrt{\left(D_{1}+D_{2}\right)\left(D_{2}+D_{3}\right)}}\right)
$$

For this two-dimensional problem, it is well-known that the survival probability asymptotically varies as $t^{-\pi / 2 \theta}[6]$. Identifying $D_{1}=D_{3}=D_{L}$ and $D_{2}=D_{\ell}$, leads to

$$
\beta_{1,1}(\mathcal{R})=\left[\frac{2}{\pi} \cos ^{-1} \frac{\mathcal{R}}{1+\mathcal{R}}\right]^{-1}
$$


where $\mathcal{R} \equiv D_{\ell} / D_{L}$. Similarly, one finds for the "chased" lamb

$$
\beta_{2,0}=\left[2-\frac{2}{\pi} \cos ^{-1} \frac{\mathcal{R}}{1+\mathcal{R}}\right]^{-1}
$$

Physically, when $\mathcal{R} \rightarrow \infty$, the motion of the lions becomes irrelevant and the trapped lamb problem maps to a diffusing particle in a fixed size absorbing domain. For this geometry, the survival probability decays exponentially in time, corresponding to $\beta_{1,1} \rightarrow \infty$ in Eq. (3a). Conversely, for the lamb at rest, $\mathcal{R} \rightarrow 0$, the survival probability is the square of the corresponding survival probability in the two-particle system so that $\beta_{1,1} \rightarrow 1$. For the chased lamb (Eq. (3b)), $\beta_{2,0}$ has the limiting values $\beta_{2,0}=1 / 2$ for $\mathcal{R} \rightarrow \infty$ and $\beta_{2,0}=1$ for $\mathcal{R} \rightarrow 0$. These two values are in accord with a direct consideration of the extreme cases of immobile lions or immobile lamb, respectively. Notice also that for $\mathcal{R}=1$ we get $\beta_{1,1}=3 / 2$ and $\beta_{2,0}=3 / 4$, results which were known previously [1].

Extending the above approach, the survival of a lamb in the presence of $N>2$ lions can be mapped onto the survival of an $N+1$-dimensional random walk which is confined within an absorbing hyper-wedge. This problem does not appear to be generally soluble, however. Numerically, there has been investigation [1] of the one-sided equal-diffusivities problem for the cases $N=3,4$, and 10 as part of an effort to understand the general behavior on $N$. This simulation reveals that the exponent $\beta_{N}$ grows slowly with $N$, with $\beta_{3}=0.91, \beta_{4} \approx 1.032 \pm 0.01$, and $\beta_{10} \approx 1.4$. (Because the case $N=4$ is close to the transition between a finite and infinite lamb lifetime, there is more numerical data and hence a greater precision in the estimate for $\beta_{4}$.) The understanding of this slow dependence of $\beta_{N}$ on $N$ is the focus of our work.

In the next section, we provide a heuristic argument for the dependence of $\beta_{N}$ on $N$, as well the behavior for $N \rightarrow \infty$. A more complete derivation of these results is given Sec. III. The general dependence of the survival probability on the ratio $\mathcal{R}=D_{\ell} / D_{L}$ is also considered. In Sec. IV, we treat the case of $N \rightarrow \infty$. A general discussion and conclusions are given in Sec. V.

\section{HEURISTIC ARGUMENTS FOR ONE-SIDED SIEGE}

First consider the trivial case of a stationary lamb, $D_{\ell}=0$. For non-interacting lions, the lamb survival probability is just the product of the survival probabilities associated with each lion-lamb pair. This immediately gives $S_{N}(t) \propto\left(t^{-1 / 2}\right)^{N}$, from which $\beta_{N}(0)=N / 2$. For this case, the relative positions of the lions do not matter in the asymptotic behavior of $S_{N}(t), i$. e., it is immaterial whether the lamb is "trapped" or "chased". When the lamb also diffuses, it is convenient to consider the survival probability in the rest frame of the lamb. Although the lions still diffuse independently, their relative motions with respect to the lamb are not independent. Therefore to determine the survival probability of a diffusing lamb, it more useful to track the position of the closest lion only. For concreteness and simplicity, suppose initially that all the lions are at the origin and the lamb is at $x_{0}>0$. A rough estimate for the location of the closest or "last" lion, $x_{+}(t)$, is provided by

$$
\int_{x_{+}(t)}^{\infty} \frac{1}{\sqrt{4 \pi D_{L} t}} e^{-x^{2} / 4 D_{L} t} d x=1 / N \text {. }
$$


This specifies that there should be one lion in the range $\left(x_{+}(t), \infty\right)$ out of an initial group of $N$ lions. By asymptotic expansion of this integral, the location of the last lion is given by

$$
x_{+}(t) \sim \sqrt{4 D_{L} t \ln N} .
$$

In the limit $N \rightarrow \infty$, a physically tractable initial condition is to have a uniform density of lions $c_{0}$ extending from $-\infty$ to 0 . In this situation, only a number $N \propto \sqrt{c_{0}^{2} D_{L} t}$ of the lions are "dangerous", i. e., are potential candidates for being closest to the lamb. Consequently, for $N \rightarrow \infty$, the leading behavior of $x_{+}(t)$ is

$$
x_{+}(t) \sim \sqrt{2 D_{L} t \ln \left(c_{0}^{2} D_{L} t\right)} \quad N \rightarrow \infty .
$$

The next step in our heuristic approach is to posit that for large $N$, the true stochastic motion of the last lion can be replaced by a continuous motion $x(t)$ with $x(t)=x_{+}(t)$, as given in Eqs. (5). Then the system reduces to a two-body problem of a diffusing lamb and an approaching absorbing boundary, whose location is $x_{+}(t)=\sqrt{A t}$. As discussed in the next section, the survival probability of a lamb adjacent to such an approaching "cliff" can be calculated analytically. This gives the exponent of the survival probability as $\beta \sim A / 16 D_{\ell}$. Substituting the appropriate value of $A$ as specified by Eqs. (5), we obtain $S_{N}(t) \sim t^{-\beta_{N}(\mathcal{R})}$ with $\beta_{N}(\mathcal{R}) \sim \ln (N \mathcal{R}) / 4 \mathcal{R}$ for finite $N$, and $S_{\infty}(t) \sim \exp \left(-\ln ^{2} t\right)$ for $N \rightarrow \infty$.

\section{ASYMPTOTIC ANALYSIS FOR ONE-SIDED SIEGE}

A more rigorous approach is to consider the survival probability in an auxiliary "deadline" problem whose asymptotic behavior turns out to give a tight lower bound for the true survival probability of the lamb. The deadline problem is defined as follows: Consider an imaginary point $x_{\text {dead }}(t)$ between the lamb and the lions which moves deterministically according to $x_{\text {dead }}(t)=\sqrt{A t}$. If the lamb crosses this line, it is considered to have died; analogously, if any of the lions overtakes the deadline, the lamb is again considered to have died. Our strategy is to determine the survival probability in this auxiliary problem, and then maximize this probability with respect to the parameter $A$. First notice that a deadline position which is proportional to $\sqrt{t}$ already optimizes the lamb survival probability with respect to other power-law motions for the deadline. That is, is $x_{\text {dead }}(t)$ were proportional to $t^{\alpha}$ with $\alpha<1 / 2$, we would asymptotically recover the behavior for the stationary deadline, which grossly overestimates the decay exponent as $\beta_{N}=(N+1) / 2$. Conversely, for faster than diffusive deadline motion, i. e., $\alpha>1 / 2$, the probability that the lamb does not hit the deadline decays as a stretched exponential [7]; therefore, this case can be also ignored. The marginal situation of $\alpha=1 / 2$ thus plays a fundamental role.

To compute the survival probability for the deadline problem, we have to solve two first-passage problems: (i) The survival of a diffusing particle in the proximity of a receding absorbing boundary, or cliff. This corresponds to a single lion, and we define the probability that a lion does not reach the cliff to be $S_{\text {lion }}(t) \propto t^{-\beta_{\operatorname{lion}}\left(D_{L}, A\right)}$. (ii) The survival of a diffusing particle in the proximity of an approaching cliff. This corresponds to the lamb, 
and the associated survival probability is defined as $S_{\text {lamb }}(t) \propto t^{-\beta_{\text {lamb }}\left(D_{\ell}, A\right)}$. The full survival probability $S_{N}(t) \propto t^{-\beta_{N}}$ is clearly the product $S_{\text {lion }}^{N}(t) S_{\text {lamb }}(t)$, so that $\beta_{N}=$ $\beta_{\text {lamb }}\left(D_{\ell}, A\right)+N \beta_{\text {lion }}\left(D_{L}, A\right)$. Once we know the exponents $\beta_{\text {lion }}$ and $\beta_{\text {lamb }}$, we optimize the decay exponent $\beta_{N}$ with respect to the amplitude $A$. By appealing to the method of the "optimal fluctuation" [8], we hypothesize that this extremal survival probability in the deadline problem gives the true asymptotic behavior.

Fortunately, the exponents $\beta_{\text {lamb }}$ and $\beta_{\text {lion }}$ have been computed in various physical [9] and mathematical studies [10] so that the full deadline problem is soluble. For completeness, however, we outline our approach, given in Ref. [7], which has the advantage of conceptual and technical simplicity. While this earlier work considered only the case of a receding cliff (relevant for the lions), the extension to the case of an approaching cliff can be derived with minimal additional effort. Let us therefore recall the steps in the computation of the survival probability for the case of the receding cliff. Consider a lion which is initially placed on the negative $x$-axis and that the cliff position is $x_{0}(t)=\sqrt{A t}$. In the long-time limit, the lion density approaches the scaling form [7]

$$
c(x, t) \sim t^{-\beta_{\text {lion }}-1 / 2} \mathcal{C}(\xi),
$$

where $\xi=1-\frac{x}{x_{0}}$ is the appropriate dimensionless distance variable and $\mathcal{C}(\xi)$ is a scaling function. The initial co-ordinate of the lion, $-\infty<x \leq x_{0}$, corresponds to $0 \leq \xi<\infty$. The power law prefactor is chosen to ensure that the survival probability decays as $t^{-\beta_{\text {lion }}}$, as defined previously.

Substituting Eq. (6) into the diffusion equation, one finds that the scaling function satisfies

$$
\frac{D_{L}}{A} \frac{d^{2} \mathcal{C}}{d \xi^{2}}+\frac{1}{2}(\xi-1) \frac{d \mathcal{C}}{d \xi}+\left(\beta_{\text {lion }}+\frac{1}{2}\right) \mathcal{C}=0
$$

Introducing the transformation

$$
\xi-1=\sqrt{\frac{2 D_{L}}{A}} \eta, \quad \mathcal{C}(\xi)=\exp \left(-\frac{\eta^{2}}{4}\right) \mathcal{D}(\eta),
$$

one finds that $\mathcal{D}(\eta)$ satisfies the parabolic cylinder equation of order $2 \beta_{\text {lion }}$ [12],

$$
\frac{d^{2} \mathcal{D}_{2 \beta_{\text {lion }}}}{d \eta^{2}}+\left[2 \beta_{\text {lion }}+\frac{1}{2}-\frac{\eta^{2}}{4}\right] \mathcal{D}_{2 \beta_{\text {lion }}}=0 .
$$

The absorbing boundary condition at the edge of the cliff implies

$$
\mathcal{D}\left(-\sqrt{A / 2 D_{L}}\right)=0
$$

On the other hand, to avoid a singular solution at $\eta=\infty$, the second boundary condition is

$$
\mathcal{D}(\eta=\infty)=0 .
$$

Mathematically, the determination of $\beta_{\text {lion }}$ and $\mathcal{D}(\eta)$ is equivalent to finding the ground state energy and wave function of a quantum particle in a potential composed of an infinite 
barrier at $\eta=-\sqrt{A / 2 D_{L}}$ and the harmonic oscillator potential for $\eta>-\sqrt{A / 2 D_{L}}$ [11]. Higher excited states do not contribute in the long time limit. This relation with quantum mechanics allows one to apply well-known techniques to determine the asymptotic behavior [7,10]. Among the two elemental solutions of the parabolic cylinder equation, $\mathcal{D}_{2 \beta_{\text {lion }}}(\eta)$ and $\mathcal{D}_{2 \beta_{\text {lion }}}(-\eta)$, only the former satisfies the boundary condition $\mathcal{D}(\infty)=0$. Therefore, the absorbing boundary condition of Eq. (10a) determines the decay exponent $\beta_{\text {lion }}=\beta_{\text {lion }}\left(D_{L}, A\right)$.

As discussed previously, the interesting behavior emerges in the large $N$ limit. For this case, the deadline position grows as $\sqrt{A t}$ but with an anomalously large amplitude $A$. Consequently, the probability distribution of each lion is only weakly affected by the receding deadline. This allows us to employ the "free particle" particle Gaussian approximation for the probability distribution of each lion. Although this form does not satisfy the absorbing boundary condition, the error is negligible because $A \gg 1$. Consequently, we can determine the decay exponent simply by computing the flux to the absorbing boundary for the assumed Gaussian probability distribution [7]. This yields

$$
\beta_{\text {lion }}\left(D_{L}, A\right) \simeq \sqrt{\frac{A}{4 \pi D_{L}}} e^{-A / 4 D_{L}}
$$

In the limit $A \rightarrow \infty$, this simple-minded approach coincides with the results from a complete analysis in terms of the parabolic cylinder function solution.

An analogous, but simpler, treatment applies for the approaching cliff, which we use to describe the interaction of the deadline with the lamb. That is, suppose that a lamb is initially placed on the positive $x$-axis and that there is an approaching cliff whose location is at $\sqrt{A t}$. To solve this problem by the same approach as the receding cliff, we introduce the appropriate dimensionless length variable $\xi=\frac{x}{x_{0}}-1$ and make the analogous scaling ansatz as in Eq. (6), so that Eq. (7) is replaced by

$$
\frac{D_{\ell}}{A} \frac{d^{2} \mathcal{C}}{d \xi^{2}}+\frac{1}{2}(\xi+1) \frac{d \mathcal{C}}{d \xi}+\left(\beta_{\text {lamb }}+\frac{1}{2}\right) \mathcal{C}=0
$$

For this case, it is again helpful to introduce $\eta$ via $\xi+1=\sqrt{\frac{2 D_{\ell}}{A}} \eta$ and $\mathcal{C}(\xi)=e^{-\eta^{2} / 4} \mathcal{D}(\eta)$. The scaling function $\mathcal{D}(\eta)$ is again the parabolic cylinder function of order $2 \beta_{\text {lamb }}$ and the absorbing boundary condition,

$$
\mathcal{D}_{2 \beta_{\text {lamb }}}\left(\sqrt{A / 2 D_{\ell}}\right)=0
$$

now determines the decay exponent $\beta_{\text {lamb }}=\beta_{\text {lamb }}\left(D_{\ell}, A\right)$. Since the relevant zero of the parabolic cylinder function $\eta=\sqrt{A / 2 D_{\ell}}$ is large, $\beta_{\text {lamb }}$ is also large. Then an inspection of Eq. (9) provides the estimate $2 \beta_{\text {lamb }}+\frac{1}{2} \simeq \frac{\eta^{2}}{4}$, or

$$
\beta_{\operatorname{lamb}}\left(D_{\ell}, A\right) \simeq \frac{A}{16 D_{\ell}} .
$$

Therefore the total decay exponent for the deadline problem is

$$
\beta_{N}(\mathcal{R}, A)=\beta_{\text {lamb }}\left(D_{\ell}, A\right)+N \beta_{\text {lion }}\left(D_{L}, A\right) \simeq \frac{A}{16 D_{\ell}}+N \sqrt{\frac{A}{4 \pi D_{L}}} e^{-A / 4 D_{L}} .
$$


Minimizing this expression with respect to $A$ yields the optimal value $A^{*} \sim 4 D_{L} \ln (4 N \mathcal{R})$. Thus the deadline motion is enhanced by a factor of $\ln N$ compared simple diffusion; notice that this coincides with the motion of the last lion in a pride of $N$ lions. Correspondingly, the optimal value of the decay exponent $\beta_{N}(\mathcal{R}) \equiv \beta_{N}\left(\mathcal{R}, A^{*}\right)$ is

$$
\beta_{N}(\mathcal{R}) \sim \frac{\ln (4 N \mathcal{R})}{4 \mathcal{R}}
$$

Our construction of the deadline problem relies on the assumption that $N \gg 1$. This assumption is crucial, otherwise the deadline problem would not provide a meaningful approximation for the behavior of the original system. However, the physical nature of the problem suggests that different asymptotic behaviors for the lamb survival probability should arise for $\mathcal{R} \gg 1$ and $\mathcal{R} \ll 1$. In fact, consideration of the limiting cases of a stationary lamb and of stationary lions, suggests that Eq. (16) is actually valid only for $N^{-1} \ll \mathcal{R} \ll \ln N$. In the slow-lamb limit, $\mathcal{R} \ll N^{-1}$, the logarithmic behavior of Eq. (16) should cross over to that of the stationary-lamb limit, namely, $\beta_{N}(0)=N / 2$. In the complementary fast-lamb limit, $\mathcal{R} \gg \ln N$, the behavior of the stationary lion case should be recovered, in which $\beta_{N}(\infty)=1 / 2$. Thus the full dependence of $\beta_{N}$ on the diffusivity ratio $\mathcal{R}$ is expected to be

$$
\beta_{N}(\mathcal{R})= \begin{cases}N / 2 & \mathcal{R} \ll 1 / N ; \\ \ln (4 N \mathcal{R}) / 4 \mathcal{R} & 1 / N \ll \mathcal{R} \ll \ln N ; \\ 1 / 2 & \mathcal{R} \gg \ln N .\end{cases}
$$

The non-universal dependence of $\beta_{N}$ on the diffusivity ratio for the intermediate regime of $1 / N \ll \mathcal{R} \ll \ln N$ is the generalization of the exponents in Eqs. (3), for the three-particle system, to arbitrary $N$.

\section{INFINITE NUMBER OF LIONS}

Consider now a lamb which is under one-sided siege by an infinite pride of lions. (These lions need to be distributed over an infinite domain so that their density is everywhere finite. If the lion density were infinite at some point, then the closest lion would move inexorably toward to the lamb at each step, leading to the survival probability decaying exponentially in time.) The interesting situation is when the lions are all to one side of the lamb. However, to introduce our approach, it is instructive to consider first the simpler two-sided problem, in which lions are uniformly and symmetrically distributed with unit density on either side of a stationary lamb, a problem has been previously investigated by asymptotic and exact methods $[13,14]$. For completeness, we describe an approach which is in the spirit of the previous section.

For a lamb at the origin, the density of the lions $c(x, t)$ may be found by solving the diffusion equation with an absorbing boundary condition at $x=0$ and with the initial condition of a unit density everywhere. This yields [5] 


$$
c_{\mathrm{lamb}}(x, t)=\frac{2}{\sqrt{\pi}} \int_{0}^{|x| / \sqrt{4 D_{L} t}} d \zeta e^{-\zeta^{2}} .
$$

Thus the diffusive flux of lions toward the lamb is $D_{L}\left(\left.\frac{\partial c}{\partial x}\right|_{x=0^{+}}-\left.\frac{\partial c}{\partial x}\right|_{x=0^{-}}\right)=\sqrt{\frac{4 D_{L}}{\pi t}}$. The survival probability $S_{\infty}(t)$ therefore obeys

$$
\frac{d S_{\infty}(t)}{d t}=-S_{\infty}(t) \sqrt{\frac{4 D_{L}}{\pi t}}
$$

with solution

$$
S_{\infty}(t)=\exp \left[-4 \sqrt{\frac{D_{L} t}{\pi}}\right] .
$$

When both the lion and lamb are diffusing, a faster decay occurs. However, since the dominant annihilation mechanism arises from the diffusive flux of lions toward the lamb, we expect that the asymptotic decay is still given by Eq. (20) [13]. The crucial feature of this two-sided problem is that there is no good "survival" strategy, so that the lamb survival probability must decay rapidly in time.

For a lamb under one-sided siege, we again attempt a solution via the auxiliary deadline model. Assume that the deadline undergoes enhanced square-root motion, $i$. e., $x_{\text {dead }}(t)=\sqrt{A t}$, with $A \gg 1$. Repeating the steps employed previously for a finite pride of lions, we have $S_{\infty}(t)=S_{\text {lion }}(t) S_{\text {lamb }}(t)$, with $S_{\text {lamb }}(t) \propto t^{-A / 16 D_{\ell}}$, as in the case of a finite pride. To determine $S_{\text {lion }}(t)$ we again use a free particle approximation, since we expect that the amplitude $A$ will be large. Thus for the probability density of the lions, we ignore the adsorbing boundary condition on the moving deadline. For the initial condition of unit density of lions for $x<0$ and zero density otherwise, the time dependent lion density is [5]

$$
c_{\text {lion }}(x, t)=\frac{1}{\sqrt{\pi}} \int_{x / \sqrt{4 D_{L} t}}^{\infty} d \zeta e^{-\zeta^{2}} .
$$

Although this solution disagrees with the adsorbing boundary condition on the deadline, the disagreement is of order $e^{-A / 4 D_{L}}$ and is negligible when $A \gg 1$.

Computing the diffusive flux of lions through the deadline, we make use of Eq. (21) and $A / D_{L} \gg 1$ to find

$$
-\left.D_{L} \frac{\partial c}{\partial x}\right|_{x=x_{\text {dead }}(t)} \simeq \sqrt{\frac{D_{L}}{4 \pi t}} e^{-A / 4 D_{L}} .
$$

The lion survival probability, $S_{\text {lion }}(t)$, therefore obeys

$$
\frac{d S_{\mathrm{lion}}(t)}{d t} \simeq-S_{\mathrm{lion}}(t) \sqrt{\frac{D_{L}}{4 \pi t}} e^{-A / 4 D_{L}},
$$

with solution

$$
S_{\text {lion }}(t) \simeq \exp \left[-e^{-A / 4 D_{L}} \sqrt{\frac{D_{L} t}{\pi}}\right]
$$


Thus the full survival probability is

$$
S_{\infty}(t) \propto \exp \left[-e^{-A / 4 D_{L}} \sqrt{\frac{D_{L} t}{\pi}}-\frac{A}{16 D_{\ell}} \ln t\right] .
$$

Maximizing this survival probability with respect to $A$, we find that optimal value, $A^{*}$, grows in time as,

$$
A^{*} \sim 2 D_{L} \ln \left[\frac{t}{\ln ^{2} t}\right] .
$$

The leading logarithmic behavior is in accord with our naive estimate given in Sec. II. Combining Eqs. (25) and (26) gives

$$
S_{\infty}(t) \propto \exp \left[-\ln ^{2} t\right]
$$

Thus we obtain a survival probability for the one-sided system which decays faster than any power law and slower than any stretched exponential. The decay is, however, universal in that the power of logarithm does not depend on the diffusivity ratio $\mathcal{R}=D_{\ell} / D_{L}$.

\section{SUMMARY AND DISCUSSION}

For a diffusing lamb in one dimension which is adjacent to a pride of $N$ diffusing, predatory lions, the survival probability of the lamb decays as $S_{N}(t) \sim t^{-\beta_{N}}$ with $\beta_{N}$ proportional to $\ln N$. This slow increase of $\beta_{N}$ on $N$ reflects the fact that the dominant contribution to the survival probability arises from realizations in which the lamb "runs away" from the lions. Consequently, each additional lion in the system has a progressively weaker effect on the lamb survival. This is in contrast to the case of a stationary lamb, where each additional lion is equally effective in hunting the lamb, so that $\beta_{N}$ is proportional to $N$. The exponent $\beta_{N}$ is also a decreasing function of the diffusivity ratio, $\mathcal{R}=D_{\ell} / D_{L}$, with $\beta_{N}=N / 2$ for $\mathcal{R}=0$ and $\beta_{N}=1 / 2$ for $\mathcal{R}=\infty$. Thus, in accord with intuition, the best survival strategy for the lamb is to diffuse faster than the lions. In contrast, for a two-sided system, where the lions initially surround the lamb, the best survival strategy for the lamb is to remain still.

The above non-universal power-law decay of $S_{N}(t)$ motivated the basic question, considered in Ref. 1, of whether the mean lamb lifetime

$$
\tau_{N} \equiv-\int_{0}^{\infty} d t t \frac{d S_{N}(t)}{d t}=\int_{0}^{\infty} d t S_{N}(t)
$$

is finite or infinite. From Eq. (28), it is clear that $\tau_{N}$ is finite for $\beta_{N}>1$, and $\tau_{N}$ diverges otherwise. The numerical evidence from Ref. 1 indicates that when $\mathcal{R}=1$ the lamb lifetime is finite for $N \geq 4$. Since our prediction for $\beta_{N}$ is anticipated to be accurate only for large $N$, we may conclude that the lamb lifetime is finite when $N \geq N^{*}(\mathcal{R})$, but cannot provide

an accurate estimate of this threshold value. Additionally, we predict that $N^{*}(\mathcal{R})$ should increase rapidly with the diffusivity ratio $D_{\ell} / D_{L}$, namely $\ln N^{*}(\mathcal{R}) \propto \mathcal{R}$ (Eq. (17)). 
For an infinite number of predators, the lamb survival probability $S_{\infty}(t)$ exhibits a $\log$-normal decay $\exp \left(-\ln ^{2} t\right)$. This contrasts sharply with the corresponding behavior in the two-sided geometry, where $S_{\infty}(t) \propto \exp \left(-t^{1 / 2}\right)$. For the one-sided geometry, it is striking that this same survival probability occurs for a reactive system consisting of a single "fast impurity" which moves with velocity $v>1$ within a semi-infinite sea of mutually annihilating ballistic particles moving at velocity $v= \pm 1$ [15]. Given the superficial similarity of the fast impurity with the lions and lamb systems, it may be interesting to seek a deeper connection between these two problems.

We close with mention of a generalization where the lions are "vicious" among themselves, in addition to stalking the lamb. (For such self-predatory lions, their number must be infinite; otherwise, the lamb survival probability has a non-zero asymptotic value.) There are two natural possibilities for the outcome when two lions meet: either (i) one lion dies (aggregation), or (ii) both die (annihilation). The first possibility is particularly simple, since the closest lion undergoes pure diffusion, independent of its individual identity. Thus the two-sided geometry reduces to the finite particle system $(M, N)=(1,1)$, with decay exponent given by Eq. (3a). The one-sided aggregation problem is even simpler since it reduces to the $(1,0)$ problem whose solution is given in Eq. (1).

Annihilating lions framework leads to more interesting behavior, as the position of the closest lion suddenly jumps away from the lamb whenever the closest two lions annihilate. The two-sided version of this problem was introduced in Ref. 16. It was found that the lamb survival probability decays as a non-universal power law, $S(t) \propto t^{-\gamma(\mathcal{R})}$, with a Smoluchowski theory predicting $\gamma(\mathcal{R})=\sqrt{(1+\mathcal{R}) / 8}$. This agrees with the obvious exact result $\gamma(1)=1 / 2$ and is close to the exact value $\gamma(0)=3 / 8$ [17]. This Smoluchowski prediction and also provides a good approximation for the simulation results for arbitrary $\mathcal{R}[16]$. Generalizations of the two-sided annihilation problems (e.g., to many dimensions) have also been discussed in [16-18]. To the best of our knowledge, however, lamb survival in the presence of a one-sided distribution of annihilating lions has not yet been treated. If one naively assumes that the two-sided death probability can be expressed in terms of independent one-sided death probabilities, then the exponents of the one-sided system, $\beta(\mathcal{R})$, and the two-sided system, $\gamma(\mathcal{R})$, are simply related by $\beta(\mathcal{R})=\gamma(\mathcal{R}) / 2$. This is clearly correct for $\mathcal{R}=0$, where the independence of the one-sided death probabilities is exact. Consequently, the known value of $\gamma(0)$ gives $\beta(0)=3 / 16$. However for $\mathcal{R}>$ 0 , the motions of the lions are not independent in the rest frame of the lamb, and the independence of one-sided killing probabilities is only an approximation. Interestingly, however, simulations suggest that for equal lion and lamb diffusivities, $\beta(\mathcal{R}=1)=1 / 4$, which conforms to the relation $\beta(\mathcal{R})=\gamma(\mathcal{R}) / 2$. We do not have an understanding of this simple yet paradoxical result. Finally, as $\mathcal{R} \rightarrow \infty$, it is clear that $\beta(\mathcal{R}) \rightarrow 1 / 2$. Thus we conclude that $\beta(\mathcal{R})$ is a slowly increasing function of $\mathcal{R}$, with $\beta(0)=3 / 16$ and $\beta(\infty)=1 / 2$.

\section{ACKNOWLEDGMENTS}

We thank D. Griffeath for helpful correspondence. We also gratefully acknowledge NSF grant DMR-9219845 and ARO grant \#DAAH04-93-G-0021 for partial support of this research. 


\section{REFERENCES}

1. M. Bramson and D. Griffeath, "Capture problems for coupled random walks", in Random walks, Brownian motion, and interacting particle systems: a festschrift in honor of Frank Spitzer, eds. R. Durrett and H. Kesten, (Boston, Birkhauser, 1991), 153-188.

2. D. ben-Avraham, "Computer simulation methods for diffusion-controlled reactions", J. Chem. Phys. 88, 941-947 (1988); M. E. Fisher and M. P. Gelfand, "The reunions of three dissimilar vicious walkers", J. Stat. Phys. 53, 175-189 (1988).

3. M. E. Fisher, "Walks, walls, wetting, and melting", J. Stat. Phys. 34, 667-729 (1984).

4. D. A. Huse and M. E. Fisher, "Commensurate melting, domain walls, and dislocations", Phys. Rev. B 29, 239-270 (1984); R. Lipowsky and T. M. Nieuwenhuizen, "Intermediate fluctuation regime for wetting transitions in two dimensions", J. Phys. A 21, L89-L94 (1988); P. J. Forrester, "Probability of survival for vicious walkers near a cliff", J. Phys. A 22, L609-L613 (1989); J. W. Essam and A. J. Guttmann, "Vicious walkers and directed polymer networks in general dimensions", Phys. Rev. E 52, 5849-5862 (1995).

5. G. H. Weiss and R. J. Rubin, "Random walks: Theory and selected applications", Adv. Chem. Phys. 52, 363-505 (1983), and references therein; see also N. G. van Kampen, Stochastic Processes in Physics and Chemistry (North-Holland, Amsterdam, 1981).

6. H. S. Carslaw and J. C. Jaeger, Conduction of Heat in Solids Chap. XI (Oxford University Press, Oxford, 1959); E. B. Dynkin and A. A. Yushkevich, Markov Processes: Theorems and Problems (Plenum Press, NY, 1969); D. L. Burkholder, "Exit times of Brownian motion, harmonic majorization, and Hardy spaces" Adv. Math. 26, 182-205 (1977); R. D. De Blassie, "Exit times from cones in $\mathbf{R}^{n}$ of Brownian motion", Z. Wahr. verb. Gebiete 74, 1-29 (1987).

7. P. L. Krapivsky and S. Redner, "Life and death in an expanding cage and at the edge of a receding cliff", Amer. J. Phys., to appear.

8. I. M. Lifshits, S. A. Gredeskul, and L. A. Pastur, Introduction to the theory of disordered systems (Wiley, New York, 1988).

9. L. Turban, "Anisotropic critical phenomena in parabolic geometries: The directed selfavoiding walk", J. Phys. A 25, L127-L134 (1992); F. Iglói, "Directed polymer inside a parabola: Exact solution", Phys. Rev. A 45, 7024-7029 (1992); F. Iglói, I. Peschel, and L. Turban, "Inhomogeneous systems with unusual critical behaviour", Adv. Phys. 42, 683-740 (1993).

10. L. Breiman, "First exit time from the square root boundary", Proc. Fifth Berkeley Symp. Math. Statist. and Probab., 2, 9-16 (1966); H. E. Daniels, "The minimum of a stationary Markov superimposed on a U-shape trend", J. Appl. Prob. 6, 399408 (1969); K. Uchiyama, "Brownian first exit from sojourn over one sided moving boundary and application", Z. Wahrsch. verw. Gebiete 54, 75-116 (1980); P. Salminen, "On the hitting time and the exit time for a Brownian motion to/from a moving boundary", Adv. Appl. Prob. 20, 411-426 (1988).

11. L. I. Schiff, Quantum Mechanics (McGraw-Hill, New York, 1968). 
12. C. M. Bender and S. A. Orszag, Advanced Mathematical Methods for Scientists and Engineers (McGraw-Hill, New York, 1978).

13. S. Redner and K. Kang, "Kinetics of the scavenger reaction", J. Phys. A 17, L451L455 (1984).

14. A. Blumen, G. Zumofen, and J. Klafter, "Target Annihilation by Random Walkers", Phys. Rev. B 30, 5379-5382 (1984).

15. P. L. Krapivsky, S. Redner, and F. Leyvraz, "Ballistic annihilation kinetics: The case of discrete velocity distributions", Phys. Rev. E 51, 3977-3987 (1995).

16. P. L. Krapivsky, E. Ben-Naim, and S. Redner, "Kinetics of heterogeneous singlespecies annihilation", Phys. Rev. E 50, 2474-2481 (1994).

17. B. Derrida, "Exponents appearing in the zero-temperature dynamics of the 1D Potts model", J. Phys. A 28, 1481-1491 (1995); B. Derrida, V. Hakim, and V. Pasquier, "Exact first-passage exponents of 1D domain growth: relation to a reaction-diffusion model", Phys. Rev. Lett. 75, 751-754 (1995).

18. J. Cardy, "Proportion of unaffected sites in a reaction-diffusion process", J. Phys. A 28, L19-L24 (1995); E. Ben-Naim, "Reaction kinetics of cluster impurities", Phys. Rev. E 53, 1566-1571 (1996); M. Howard, "Fluctuation Kinetics in a Multispecies Reaction-Diffusion System", cond-mat 9510053. 